\title{
Cecryphalini Yakovlev et Witt, trib. n. - new tribe of Carpenter- Moths (Lepidoptera: Cossidae: Zeuzerinae)
}

\section{Cecryphalini Yakovlev et Witt, trib. n. - новая триба древоточџев (Lepidoptera: Cossidae: Zeuzerinae)}

\author{
R.V. Yakovlev ${ }^{1,2}$, G.G. Sokolova ${ }^{1}$, Th. Witt ${ }^{3}$ \\ Р.В. Яковлев ${ }^{1,2}$, Г.Г. Соколова ${ }^{1}$, Т. Витт ${ }^{3}$
}

\footnotetext{
${ }^{1}$ Altai State University, Lenina pr. 61, RF-656049, Barnaul, Russia.

${ }^{2}$ Tomsk State University, Laboratory of Biodiversity and Ecology, Lenina pr. 36, 634050 Tomsk, Russia E-mail: yakovlev_asu@mail.ru

${ }^{3}$ Museum Witt, Tengstrasse 33, D-80796, Munich, Germany. E-mail: thomas@witt-thomas.com

1 Алтайский государственный университет, пр. Ленина 61, Барнаул, 656049, Россия.

${ }^{2}$ Томский государственный университет, Лаборатория биоразнообразия и экологии, пр. Ленина 33, Томск, 634050, Россия.
}

KEYWORDS: Lepidoptera, Cossidae, Cecryphalini, new tribus, new synonym, catalog.

КЛЮЧЕВЫЕ СЛОВА: Lepidoptera, Cossidae, Cecryphalini, новая триба, новый синоим, каталог.

ABSTRACT. A new tribe, Cecryphalini Yakovlev et Witt, trib.n., is proposed to includes two genera, Cecryphalus Schoorl, 1990 and Tarsozeuzera Schoorl, 1990 and their included species. A new synonimy Zeuzera strix Grum-Grshimailo, 1853 = Zeuzera nubila Staudinger, 1895, syn.n. is established; a redescription of the genus Tarsozeuzera is presented. The illustrated catalog of the eight species of the tribe, listing type specimens is provided. The distribution of all the known species is shown on range maps.

РЕЗЮМЕ. В статье приводится описание новой трибы Cecryphalini Yakovlev et Witt, trib.n., включающей два рода: Cecryphalus Schoorl, 1990 и Tarsozeuzera Schoorl, 1990 и восемь видов. Установлена новая синонимия Zeuzera strix Grum-Grshimailo, 1853 = Zeuzera nubila Staudinger, 1895, syn.n.; переописан род Tarsozeuzera. Дан иллюстрированный (в том числе типовыми экземплярами) каталог трибы. Даны карты распространения всех известных видов трибы.

\section{Introduction}

Cossidae (Lepidoptera) is a family including over 1000 taxa of the species group [Nieukerken et al., 2011]. The family system is still not sufficiently studied; in particular, tribes have been not erected. At present, taking into account the complete morphological study of the genera, it is possible to begin the work on the supergeneric taxonomy of the family.

I.V. Kozhantshikov in an unpublished 1950 manuscript was the first to place the Palaearctic species
Zeuzera nubila Staudinger, 1895 and Z. helenae Le Cerf, 1924 into a single genus. In this manuscript on Cossidae of the USSR (found in the archives of the Lepidopterological Laboratory of the Zoological Institute of the Russian Academy of Science), he suggested the generic name Semizeuzera for these two species, which is unavailable (Fig. 1). Also, Kozhantshikov noted a significant external similarity of $Z$. nubila and $Z$. helenae to the tropical species: Z. kochi Semper, 18961902 and Duomitus fuscipars Hampson, 1892.

Schoorl [1990] described two Zeuzerinae genera clearly distinguished from the others: Cecryphalus Schoorl, 1990 (type species — Zeuzera nubila Staudinger, 1895) and Tarsozeuzera Schoorl, 1990 (type species - Zeuzera kochi Semper, 1902). Schoorl [1990: 161, fig. 75]

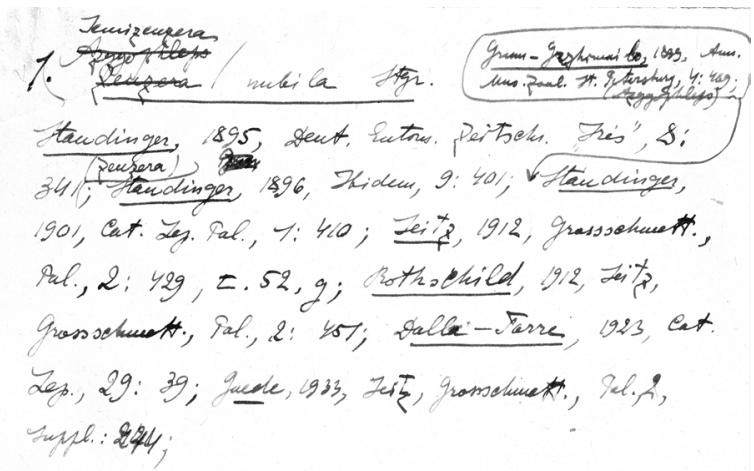

Fig. 1. Fragment of Kozhantshikov's manuscript [from Yakovlev, 2011a].

Рис. 1. Фрагмент рукописи И. Кожанчикова [из Яковлев, 2011a]

How to cite this article: Yakovlev R.V., Sokolova G.G., Witt Th. 2018. Cecryphalini Yakovlev et Witt, trib. n. — new tribe of Carpenter-Moths (Lepidoptera: Cossidae: Zeuzerinae) // Russian Entomol. J. Vol.27. No.4 P.415424. doi: 10.15298/rusentj.27.4.09 
noticed the great similarity of these genera and united them to one clade. Unfortunately, he did not provide a diagnoses basing on the genitalic characteristics.

The redescription of Cecryphalus was published by Yakovlev [2011a]. Additionally, four new species of the Tarsozeuzera from Central and South Africa, Thailand, and New Guinea were described by Yakovlev [2006, 2011b].

The examination of genitalic structures of representatives of all the Zeuzerinae genera of the World fauna, we have determined that the genera Cecryphalus and Tarsozeuzera should be included in a new tribe.

\section{Material and methods}

In the study, the materials from the following collections were examined:

AHU - collection of Armin Hauenstein (Untermünkheim, Germany);

BMNH - Natural History Museum (London, G.B.);

$\mathrm{CMNH}$ - Carnegie Museum of Natural History (Pittsburg, USA);

MNHN - Muséum National d'Histoire Naturelle (Paris, France);

MNKB - Museum für Naturkunde, Leibniz Institut für Evolution und Biodiversitatsforschung, (Berlin, Germany);

MRAC - Museum Royal of Central Africa (Tervuren, Belgium);
MSW — collection of Manfred Ströhle (Weiden, Germany);

MWM - Museum of Thomas Witt (Munich, Germany);

RMM - collection of Raymond Murphy (Mzuzu, Malawi);

RYB - collection of Roman Yakovlev (Barnaul, Russia);

ZISP - Zoological Institute of Science Academy of Russian Federation (Sankt-Petersburg, Russia);

ZMKU - Zoological Museum at Kiev State University (Kiev, Ukrainia);

ZMMU - Zoological Museum of Moscow University (Moscow, Russia);

ZSM - Zoologische Staatssammlung der Bayerischen Staaten (Munich, Germany).

Genitalia slides were examined with a Zeiss Stemi $2000 \mathrm{C}$ microscope and images were taken with the Olympus XC 50 camera.

\section{Results}

Cecryphalini Yakovlev et Witt, trib.n.

TYPE GENUS - Cecryphalus Schoorl, 1990.

DIAGNOSIS. Presence of a noticeable notch on the outer edge (at the anal angle) of the male hind wing, the flat apex of the uncus.

DESCRIPTION. Male. Medium size. Antenna typical of Zeuzerinae: basally bipectinate, distally simple. Fore wing narrow, long, apex sharp. Hind wing short, apex rounded,
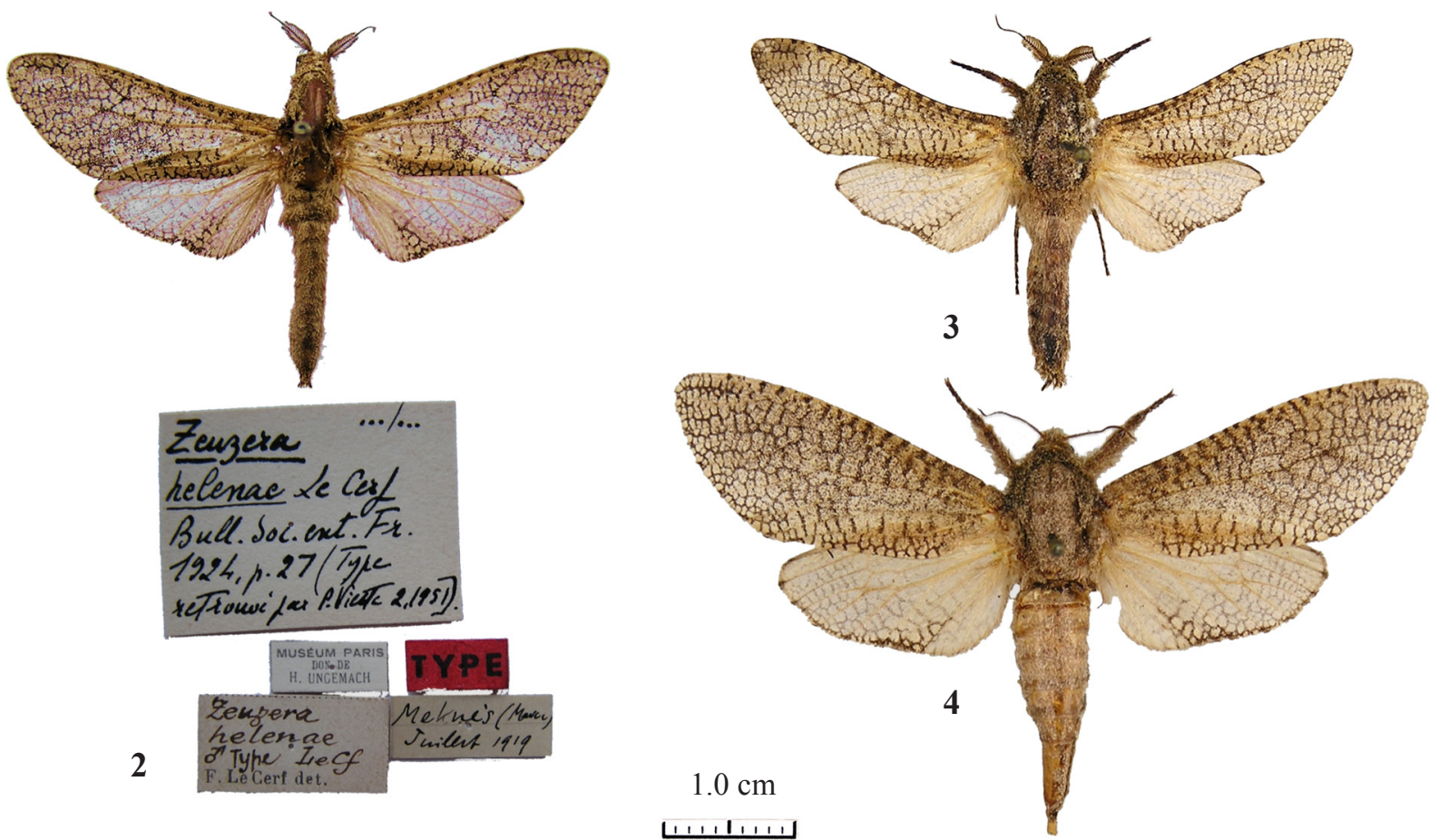

Figs 2-4. Cecryphalus (adult specimens): $2-$ C. helenae, O (holotype of Zeuzera helenae Le Cerf, 1924) (MNHN); 3 - C. helenae, $\mathrm{O}^{7}$, Algier, El Mesrane, Predota, 12.7.1931 (MWM); 4-C. helenae, +, Algier, El Mesrane, Predota, 12.7.1931 (MWM).

Рис. 2-4. Cecryphalus (имаго): 2 - C. helenae, О (голотип Zeuzera helenae Le Cerf, 1924) (MNHN); 3 - C. helenae, O', Algier, El Mesrane, Predota, 12.7.1931 (MWM); $4-$ C. helenae,, , Algier, El Mesrane, Predota, 12.7.1931 (MWM). 

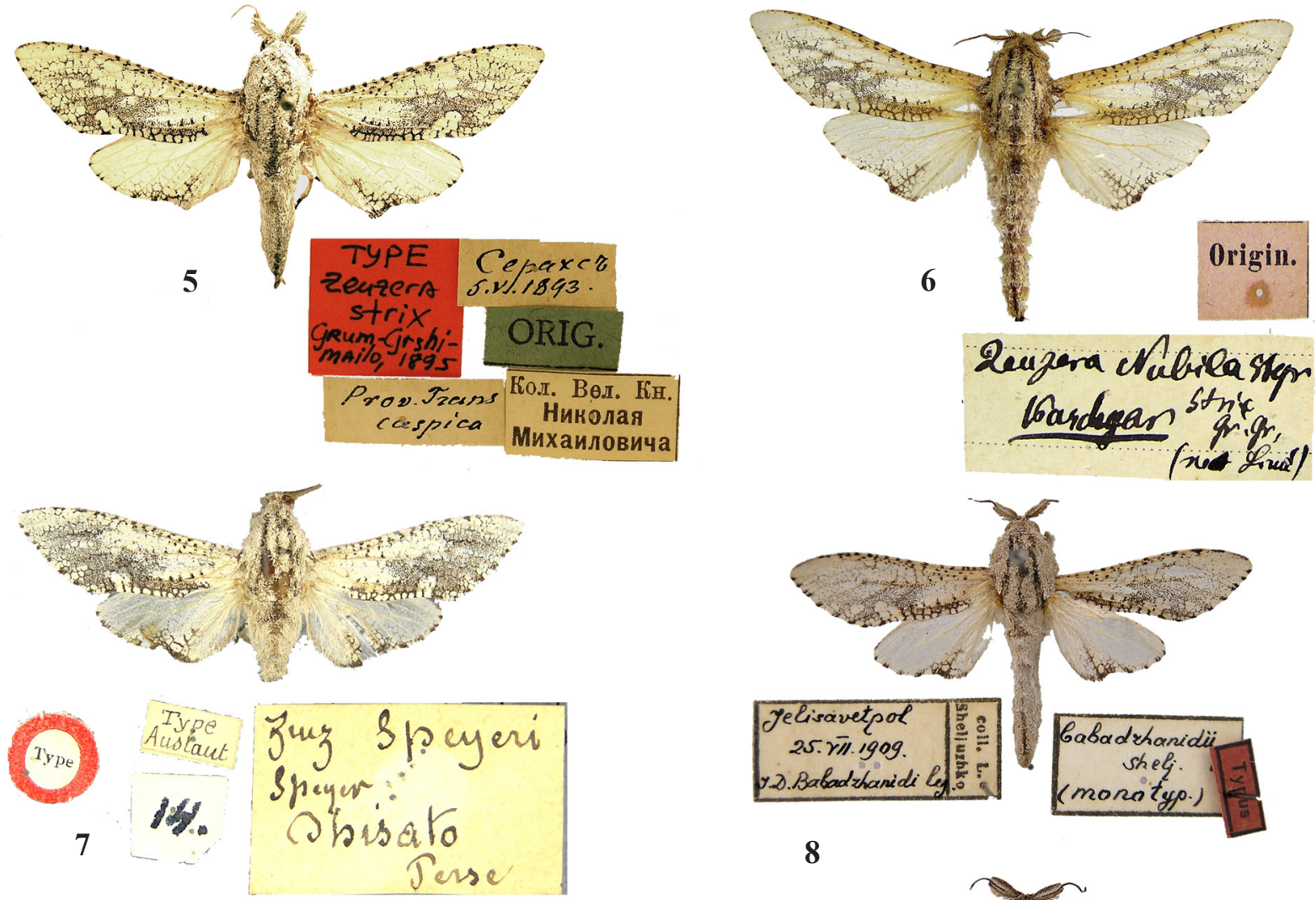

8
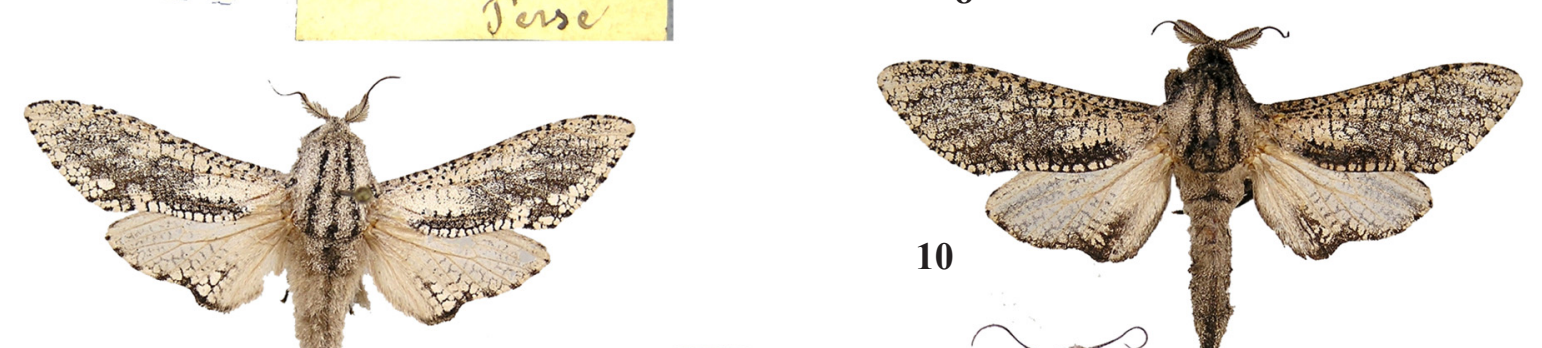

9
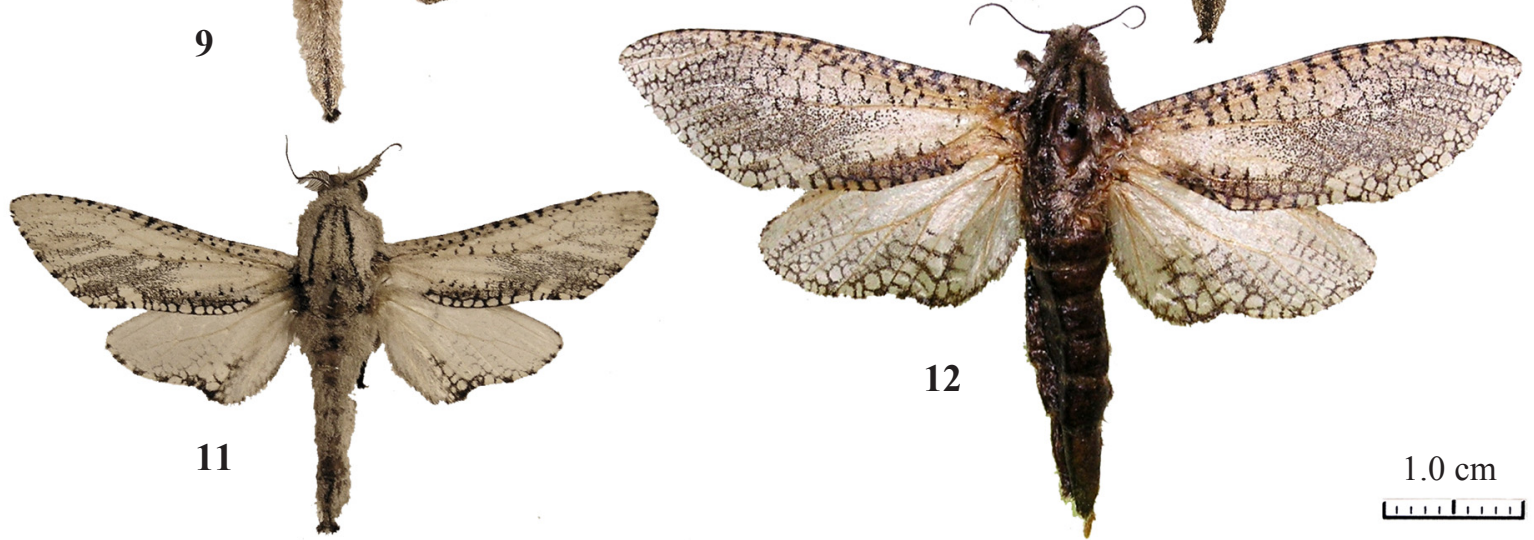

Figs 5-12. Cecryphalus (adult specimens): 5 - C. strix, O' (holotype of Zeuzera strix Grum-Grshimailo, 1895) (ZISP); 6 - C. strix, $\sigma^{7}$ (cotype of Zeuzera nubila Staudinger, 1895) (MNKB); $7-$ C. strix, $\sigma^{77}$ (holotype of Zeuzera speyeri Austaut, 1897) (BMNH); $8-C$. strix, ( (holotype of Zeuzera nubila babadzhanidii Sheljuzhko, 1913) (ZMKU); 9 - C. strix, O', Kyrgyzstan, Issyk-Kul Lake, $10 \mathrm{~km} \mathrm{~W} \mathrm{of}$ Tasor, 10-11.07.1994, leg. Spatenka (MWM); $10-$ C. strix, O , Kazakhstan, S Balkhash Lake, vil. Topar, 10.07.1986, leg. A. Kondratiev (MWM); $11-$ C. strix, $\sigma^{\prime}$, [NW China], Shamil, Hami (MNKB); $12-$ C. strix,, , SW Tajikistan, Pjandzh River, Tigrovaja Balka natural reservation, 1.08.2000, leg. V. Perepechaenko (RYB).

Рис. 5-12. Cecryphalus (имаго): 5 - C. strix, О (голотип Zeuzera strix Grum-Grshimailo, 1895) (ZISP); 6 - C. strix, О' (котип Zeuzera nubila Staudinger, 1895) (MNKB); 7 - C. strix, Оフ (голотип Zeuzera speyeri Austaut, 1897) (BMNH); 8 - C. strix, 9 (голотип Zeuzera nubila babadzhanidii Sheljuzhko, 1913) (ZMKU); 9 - C. strix, O’, Kyrgyzstan, Issyk-Kul Lake, 10 km W of Tasor, 10-11.07.1994, leg. Spatenka (MWM); $10-$ C. strix, Oح, Kazakhstan, S Balkhash Lake, vil. Topar, 10.07.1986, leg. A. Kondratiev (MWM); $11-C$. strix, $\mathrm{O}^{7}$, [NW China], Shamil, Hami (MNKB); 12 - C. strix,, , SW Tajikistan, Pjandzh River, Tigrovaja Balka natural reservation, 1.08.2000, leg. V. Perepechaenko (RYB). 
notch on outer edge (at anal angle). Wings grey, brownish, with fine reticulated pattern.

Male genitalia. Uncus short, apex flat; gnathos arms short, ribbon-like, not fused; valve simple, narrow, apex rounded; juxta saddle-like, with leaf-like lateral processes, directed dorsally; saccus poorly expressed, semi-circular; phallus thick, relatively short, with longitudinal folds, vesica with portions of scabination, cornutus robust, equal to $1 / 2$ of phallus in length.

Female larger than male. Antenna filiform. Wings slightly wider, hind wing without notch. Wing pattern resembles to that of male.

Female genitalia (described on the unique studied species, C. nubila). Ovipositor long. Papilla analis lanceolate, with transverse folds, posterior apophyses longer than anterior ones, ostium small, oval, slightly submerged, ductus of medium diameter, with longitudinal folds, bursa bag-like, with signum on lateral surface, ductus seminalis coming out of bursa in its medium third laterally, small bulla on long thin strand coming out of bursa basally.

Includes two genera: Cecryphalus Schoorl, 1990 and Tarsozeuzera Schoorl, 1990.

\section{Genus Tarsozeuzera Schoorl, 1990}

Schoorl, 1990: 157-159.

TYPE SPECIES - Zeuzera kochi Semper, 1896-1902 (by original designation).

REDESCRIPTION. Male. Medium size. Antenna basally bipectinate, distally simple. Thorax and abdomen densely covered with dark scales, tegula and patagium pale. Fore wing narrow, long, apex sharp. Dark field in discal area, reticulated pattern along hind and outer edges. Hind wing short, apex rounded, notch on outer edge (an anal angle), dark, fine reticulated pattern along outer edge and at anal angle.

Male genitalia. Uncus elongated, apex flat; tegumen of medium size; gnathos arms basally wide, distally narrowing, short, not fused; valve leaf-like, edges practically even, apex rounded; juxta saddle-like, with leaf-like lateral processes, directed dorsally; saccus poorly expressed, semicircular; phallus thick, short, slightly bent, with longitudinal folds (more expressed in distal half), vesica with portions of scabination, cornutus robust, equal to $1 / 2$ of phallus in length.

The genus includes 6 species.

DIAGNOSIS. The genus is most similar to Cecryphalus Schoorl, 1990 from which it differs by the wide dark field in the discal area of the fore wing and by the more elongated uncus of the male genitalia.

DISTRIBUTION. Tropical regions of the Old World (Hindustan, Indochina, Southern China, Philippines, New Guinea, Africa from Congo to South Africa).

\section{Catalog of Cecryphalini Yakovlev et Witt, trib.n. Genus Cecryphalus Schoorl, 1990 \\ Schoorl, 1990: 156-157.}

TYPE SPECIES - Zeuzera nubila Staudinger, 1895 (by original designation).

\section{Cecryphalus helenae (Le Cerf, 1924)}

Figs 2-4, 25, 31.

Zeuzera helenae Le Cerf, 1924: 27.

TYPE LOCALITY: ? Marrakech (Morocco).

TYPE MATERIAL (holotype) in MNHN, examined.

DISTRIBUTION: Morocco [Rungs, 1942, 1979; de Frei-

na, Witt, 1990], Algeria.

HOST: Tamarix [Rungs, 1979].

MATERIAL EXAMINED: $7 \sigma^{7} \sigma^{7}$, Marocco, Ait-ou-Mribete, 1-14.09.1999, leg. Seibald (MSW, MWM); 1 9, Algier, Rophe de Sel, Predota, 4.7.1931 (ZSM); $3 \sigma^{7} \sigma^{7}, 2$ 우, Alg., El Mesrane, 1.7.1931, lg. Predota (ZSM); $3 \sigma^{7} \sigma^{7}$, N. Morocco, Rif Mts., Taou-

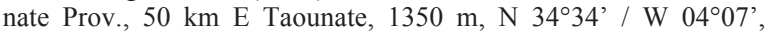
24.05.2012, leg. P. Romantsov (RYB).

\section{Cecryphalus strix (Grum-Grshimailo, 1895)}

Figs 5-12, 26-27, 31.

Zeuzera strix Grum-Grshimailo, 1895: 292.

TYPE LOCALITY: provincia Transcaspica ad urbem Serachs [Turkmenistan, Serags or NE Iran, Sarachs]. Distance betveen Serags and Sarachas $-5 \mathrm{~km}$.

TYPE MATERIAL (holotype) in ZISP, examined.

SYNONYMY:

= Zeuzera nubila Staudinger, 1895: 341 .

Type locality: Kaschgar (NW China, Tura)

Type material (syntypes) in MNKB, examined.

= Zeuzera speyeri Austaut, 1897: 45

Type locality: la Perse à Dhisato (? Uzbekistan, Dzhizak)

Type material (holotype) in BMNH, examined.

= Zeuzera nubila babadzhanidii Sheljuzhko, 1913: 21.

TYPE LOCALITY: Jelisavetpol (Gäncä, Azerbaijan).

TYPE MATERIAL (holotype by monotypy) in ZMKU, examined.

DISTRIBUTION: S. Kazakhstan, Kirgiziya, S. Mongolia, Uzbekistan, Tadzhikistan, Azerbaijan, S. Armenia, Turkmenistan, N. Iran, China (Xinjiang) [Seitz, 1912; Dalla Torre, 1923; Daniel, 1969, 1970; Hua et al., 1990; Yakovlev, 2004a, c, 2015; Yakovlev, Dubatolov, 2013; Yakovlev, Witt, 2016].

MATERIAL EXAMINED: China: $2 \sigma^{\top} \sigma^{\top}$ (syntypes) Kaschgar (MNKB); $1 \sigma^{\top}$, Shamil, Hami (MNKB). Mongolia: Reported by Daniel for: "Sudgobi aimak, $100 \mathrm{~km}$ W vom Grenzposten Ovot Chuural, 22 km W von Sajryn chudag, 1250 m, 22.6.1967; Bajanchongor aimak, Grezposten Caganbulag im Gebirge Cagan Bogd ul, 1550 m, 24.06.1967; Oase Echin gol, ca. 90 km NO vom Grenzposten Caganbulag, 950 m, 27-28.6.1967" [Daniel, 1970]; $2 \sigma^{7} \sigma^{7}, \mathrm{SW}$ Mongolia, Gobi-Altai aimak, Dzhungarian Gobi, Alag-Nuur lake, 9-10.07.2010, $1300 \mathrm{~m}, 4^{\circ} 09^{\prime} \mathrm{N}$; 94³0'E; R. Yakovlev, E. Guskova (RYB). Kazakhstan: $2 \sigma^{\top} \sigma^{\top}$, SE Kazakhstan, near Koktal,

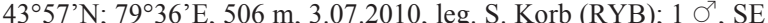
Kazakhstan, Ili Valley, near Panfilov, N4358'; E79³5', 600 m, 4.07.2010, leg. S. Korb (RYB); $2 \sigma^{\top} \sigma^{7}$, Alma-Ata reg., Chundzha, 26-28.06.1990, I. Pljustch (DEIM); 1 ऽ', Ili River, Topar vill., 10.07.1986, A. Kondratiev (MSW); $1 \sigma^{7}$, Sharyn riverside, Sarytogaj, on light $43^{\circ} 35^{\prime} \mathrm{N} 79^{\circ} 18^{\prime} \mathrm{E}, 01.07 .1994$ D. Milko leg. (IBBK); $1 \mathrm{~m}$, Taldy-Kurgan reg., Panfilov distr., Aidarly vill (ZISP); $1 \mathrm{~m}$, Alma-Ata reg., Balkhash distr., Ak-Kol', 7.7.1952 V. Parfent'ev

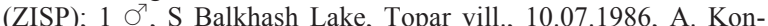
dratiev (MWM); $2 \sigma^{\top} \sigma^{7}, 50 \mathrm{~km}$ SW Dzharkent, at Ili River, 20.07.1994, leg. Kostjuk (MWM); $3 \sigma^{7} \sigma^{7}$, Almaty prov., $22 \mathrm{~km} \mathrm{~N}$ Masak, 660 m, 4345N, 78²7E, 27.07.1995, leg. Fabian (MWM); 3 O $^{7}$, Chunja, 8.07.1994, leg. Kostjuk (MWM); 1 ơ, TaldyKurgan Reg., Ili, Borokhudshir, 500 m, 19.06.1996, leg. Lukhtanov (MWM); $1 \sigma^{7}$, to S. of Balhash lake, Topar vil., Ili r., 10.07.1986,

Рис. 13-24. Tarsozeuera (имаго): 13 - T. fuscipars, О (голотип Duomitus fuscipars Hampson, 1892); 14 - T. fuscipars, O', India, Darjeeling, 5 km N Rambi, 900 m, 20"30.07.1990, leg. W. Thomas (MWM); 15 - T. fuscipars, O', Thailand, Changwat Nan, 15 km N of Bo Luang, 1000m, 7.04. 1998, leg. Csovari \& Steger (MWM); 16 - T. kochi, , (котип Zeuzera kochi Semper, 1896); 17 - T. kochi, O', Philippinen, Negros, Mt. Kanlaon, 03.1997 (MWM); 18 - T. kochi, , , Philippinen, Negros, Mt. Canlaon, W-Route via Mambucal, 600 m, 10.1996 (MWM); 19 - T. livingstoni, О७ (голотип) (MNHN); $20-$ T. livingstoni, О7, (голотип (sic!) Tacticista (n.g.) subhyalinata Clench), Cameroon (Efulen) $(\mathrm{CMNH}) ; 21$ - T. livingstoni, , Chimaliro FR, Kasungu distr., C. Malawi, $4200 \mathrm{ft} ., 31.01 .2005$, leg. R.J. Murphy (RMM); 22 - T. miklukhomaklayi (голотип) (BMNH); 23 - T. ustjuzhanini (голотип) (MWM); 24 - T. vavizola (голотип) (ZSM). 


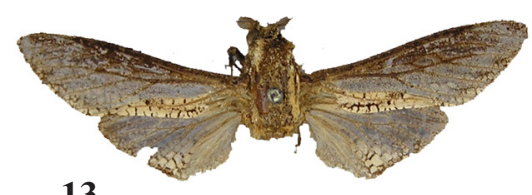

13
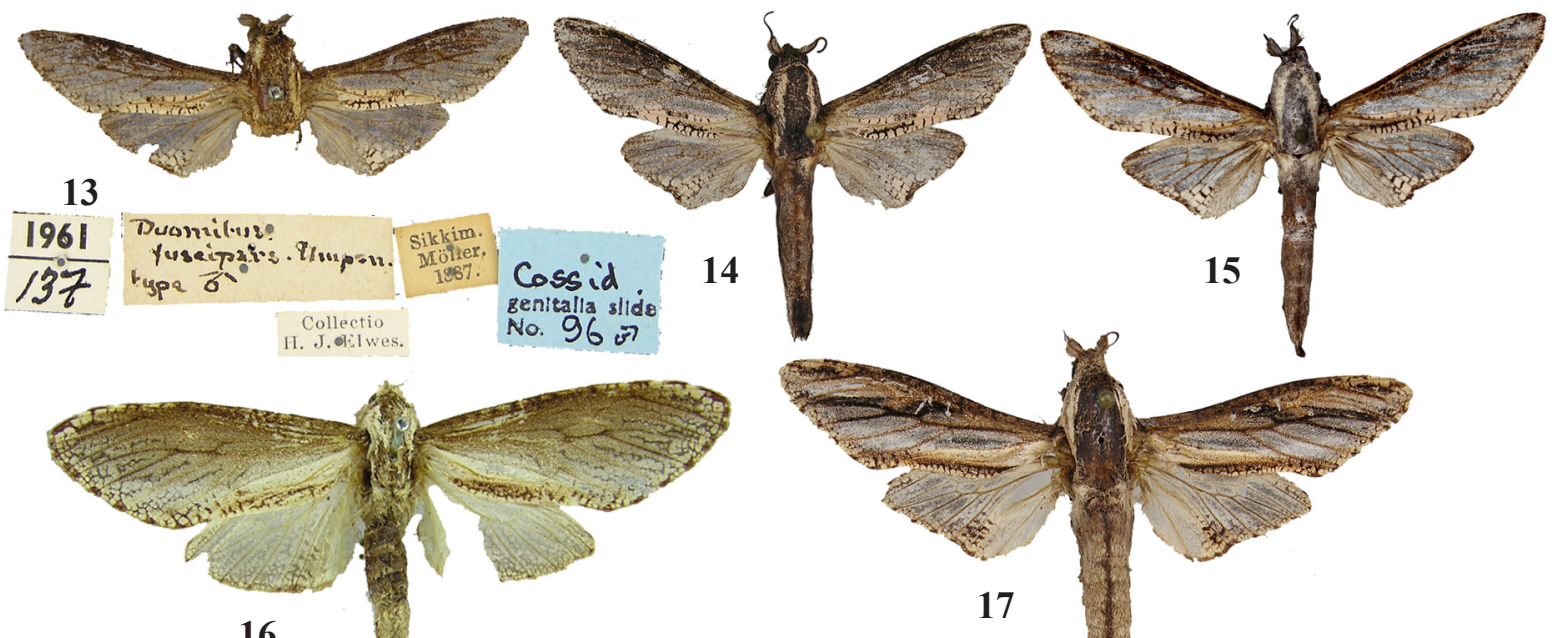

16

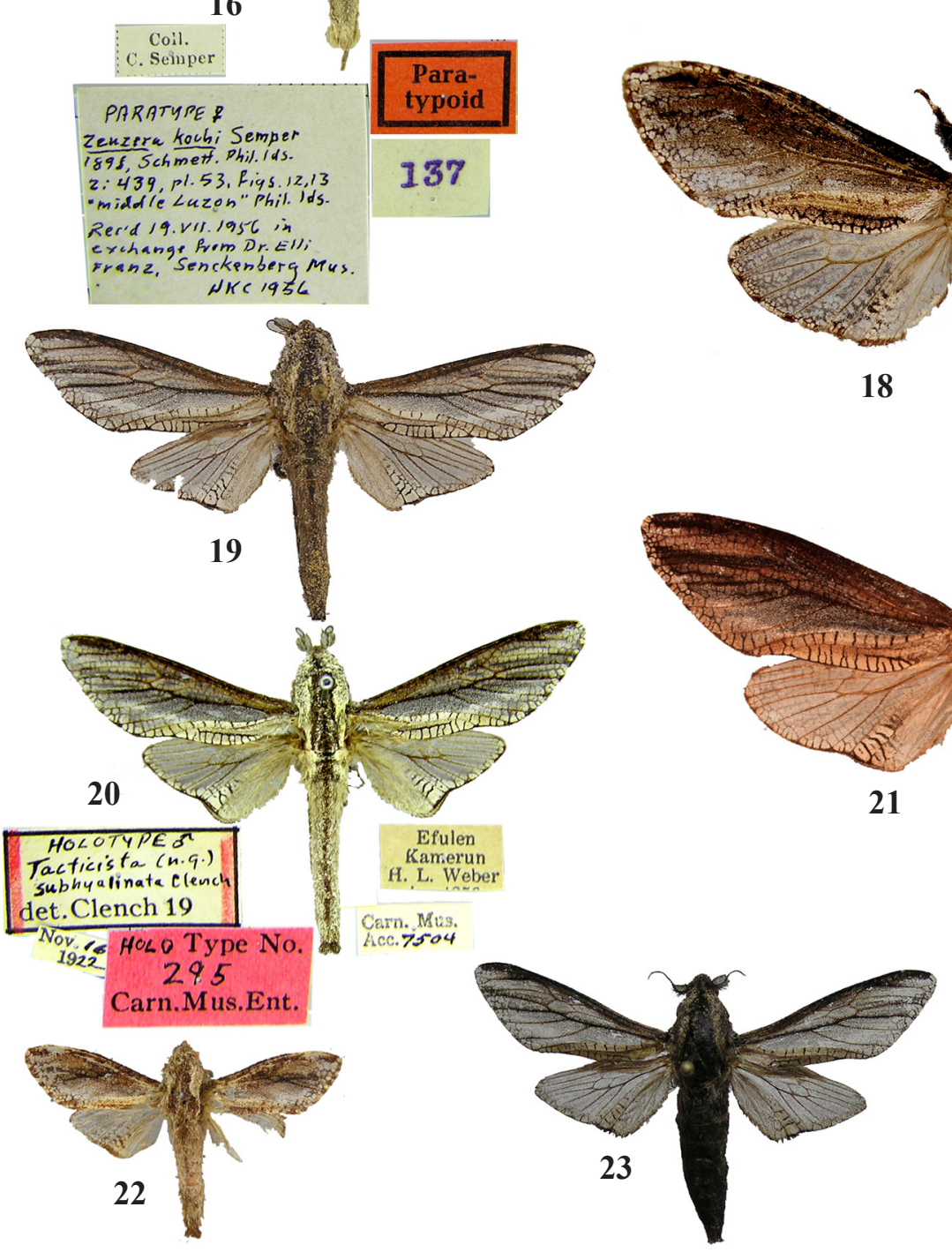

17

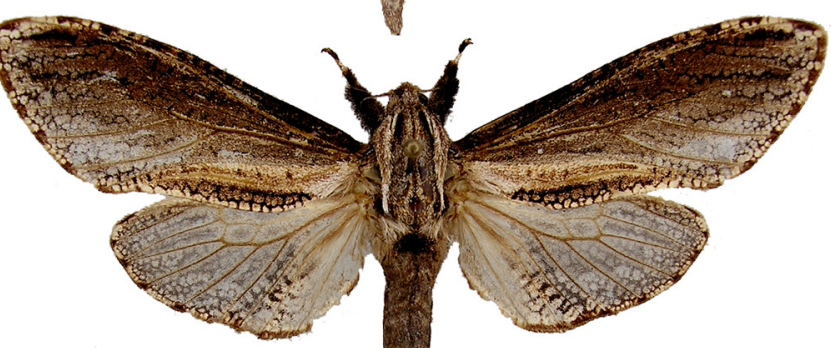

18

Figs 13-24. Tarsozeuera (adult specimens): 13 - T. fuscipars, ơ (holotype of Duomitus fuscipars Hampson, 1892); 14 - T. fuscipars, $\bigcirc^{7}$, India, Darjeeling, $5 \mathrm{~km} \mathrm{~N}$ Rambi, $900 \mathrm{~m}, 20 " 30.07 .1990$, leg. W. Thomas (MWM); $15-$ T. fuscipars, $\bigcirc^{7}$, Thailand, Changwat Nan, $15 \mathrm{~km}$ $\mathrm{N}$ of Bo Luang, 1000m, 7.04. 1998, leg. Csovari \& Steger (MWM); 16 — T. kochi, , (cotype of Zeuzera kochi Semper, 1896); 17 — T. kochi, $\mathrm{O}^{7}$, Philippinen, Negros, Mt. Kanlaon, 03.1997 (MWM); 18 - T. kochi,, , Philippinen, Negros, Mt. Canlaon, W-Route via Mambucal, 600 m, 10.1996 (MWM); 19 - T. livingstoni, Oج (holotype) (MNHN); 20 - T. livingstoni, O', (holotype (sic!) of Tacticista (n.g.) subhyalinata Clench), Cameroon (Efulen) $(\mathrm{CMNH}) ; 21$ - T. livingstoni, , Chimaliro FR, Kasungu distr., C. Malawi, $4200 \mathrm{ft} ., 31.01 .2005$, leg. R.J. Murphy (RMM); $22-T$. miklukhomaklayi (holotype) (BMNH); $23-T$. ustjuzhanini (holotype) (MWM); $24-T$. vavizola (holotype) (ZSM). 
leg. A. Kondratiev (MWM); $90^{\top} \odot^{\top}$, Kazakhstan, Almaty Region, Panfilov District, Koktal county near Akzhazyk village, Koybin River, $900 \mathrm{~m}, 44^{\circ} 10^{\prime} 11^{\prime \prime N}, 79^{\circ} 31^{\prime} 01$ "E, 511.07.2015, leg. S. Legotin, S. Didenko, M. Elistratov (RYB); $2 O^{7} \sigma^{7}$, Kazakhstan, Almaty Region, Uyghur District, $1200 \mathrm{~m}$ to EES from the bridge across Ili River, turanga forest on the sand barkhans, $505 \mathrm{~m}, 43^{\circ} 58^{\circ} 06^{\prime \prime} \mathrm{N}$ 79³7'27"E, 30.061.07.2016, leg. S. Legotin, S. Didenko, M. Elistratov (RYB); $1 \sigma^{\top}$, Kazakhstan, Almaty Region, Panfilov District, Koktal county near Akzhazyk vill., Koybin River, 1,5 km downstream from the intersection with the highway A353, $900 \mathrm{~m}$, $44^{\circ} 10^{\prime} 11^{\prime \prime N}, 79^{\circ} 31^{\prime} 01 " \mathrm{E}, 34.07 .2016$, leg. S. Legotin, S. Didenko, M. Elistratov (RYB); $1 \mathrm{O}^{7}$, Almaty Province, $9 \mathrm{~km} \mathrm{~S}$ of Konyrolen,

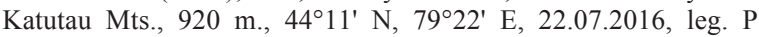
Gorbunov (RYB); $2 \sigma^{7} \sigma^{7}$, Almaty Province, $12 \mathrm{~km} \mathrm{SW}$ of Akzhar vill., Ili River Valley, 445' ${ }^{\prime} \mathrm{N}, 7^{\circ} 53^{\prime}$ E, 1.07.2017, leg. P. Gorbunov (RYB); $2 \mathrm{O}^{7} \mathrm{O}^{7}$, Jambul Province, $4 \mathrm{~km} \mathrm{SW}$ of Zhaster r./ w. station, saksaul desert, $45^{\circ} 32^{\prime} \mathrm{N}, 73^{\circ} 25^{\prime} \mathrm{E}, 30.06 .2017$, leg. P. Gorbunov (RYB); $1 \mathrm{O}^{7}, 1$ +, Kzyl-Orda reg., Aral Lake, Barsakelmes, 20.07.1979, leg. Vl. Vasilenko (RYB). Kyrgyzstan: Kyrgyzstan, Issyk-Kul Lake, $10 \mathrm{~km} \mathrm{~W}$ of Tasor, 10-11.07.1994, leg. Spatenka (MWM). Tajikistan: $4 \sigma^{7} \sigma^{7}$, Tigrovaya Balka (MSW); $2 \sigma^{7} \sigma^{7}$, Ajwadi, 350 m, 15-24.07.1963, leg. Stchetkin (AHU); $10^{7}, 22$ km SSE Kulyab, near Pistamazor vill., 1200 m, 28.07.2015, O. Pak (RYB); $60^{\top} \mathrm{O}^{\top}, 1$, , SW Tajikistan, Pjandzh River, Tigrovaja Balka natural reservation, 1.08.2000, leg. V. Perepechaenko (RYB); $10^{7}$, Tajikistan, Khatlon reg., Shurabad distr., $10 \mathrm{~km} \mathrm{~S} \mathrm{Kuljab,} \mathrm{near}$ Tugmagi vill., 900 m, 5.08.2008, leg. O. Pak \& E. Ivanova (RYB); $3 \mathrm{O}^{\top} \mathrm{O}^{\top}$, Tadzhikistan, Karatau Mt., Parchar, 830 m, 17.09.1999, leg. Yu. Stchetkin (RYB); $31 \mathrm{O}^{7} \mathrm{O}^{7}$, Tigrovaya Balka, 1-5.08.2006, leg. V. Gurko (MWM). Uzbekistan: $20^{7} \sigma^{7}$, Termez (ZISP); $20^{7} \mathrm{O}^{7}$ Baigakum, Syr-Dar'ja (ZISP); $1 \sigma^{\top}$ (holotype of Zeuzera speyeri Austaut, 1897), Dshisato [Dzhizak] (BMNH). Turkmenistan: $10^{7}$ Kara-Kala, 5.93, leg. Naglis (MSW); $20^{7} \sigma^{7}$, Kopetdagh, Sumbar riv., Tersakan, 19.07.1996, leg. Miatleusky (MSW); $3 \sigma^{7} \sigma^{7}$, Kopetdagh, Modzukly Mt., 1000 m., 18.04.1996, leg. Miatleusky (MSW); $1 \sigma^{7}$, Molla-Kara, near Krasnovodsk (ZISP); $12 \sigma^{7} \sigma^{7}$, Aj-Dare, SyrDarja (ZISP); $20^{\top} \sigma^{\top}$, Ashabad (ZISP). Azerbaijan: 1 t (holotype of Zeuzera nubila babadzhanidii Sheljuzhko, 1913), Jelisavetpol, 25.07.1909, leg. Y.D. Babadzhanidi (ZMKU). Armenia: 1 \%", Vank, 8.06.2010, leg. Yu. Shevnin (RYB)

TAXONOMICAL NOTES. The publication date in Horae Soc. Entomol. Ross. 1895, T.29: pages 1302 is April 30, 1895 (with the description of Zeuzera strix Grum-Grshimai1o, 1895: 292), and the publication date in Deutsche Entomologische Zeitschrift Iris 1895 Bd.8, Hf.2 (S.229387) is January 02, 1896 (with the description of Zeuzera nubila Staudinger, [1895]: 341). Thus, Zeuzera strix Grum-Grshimailo, 1895 = Zeuzera nubila Staudinger, [1895], new junior subjective synonym.

\section{Genus Tarsozeuzera Schoorl, 1990}

Schoorl, 1990: 157-159.

TYPE SPECIES - Zeuzera kochi Semper, 1896 (by origina designation)

\section{Tarsozeuzera fuscipars (Hampson, 1892)}

Figs 13-15, 28, 32.

Duomitus fuscipars Hampson, 1892: 309.

TYPE LOCALITY: Sikhim (India, Sikkim). ined.

TYPE MATERIAL (holotype by monotypy) in BMNH, exam-

SYNONYMY:

= Xyleutes xanthitarsus Hua, Chou, Fang et Chen, 1990: 110-111.

Type locality: Manghuai, Yunxian, Yunnan.

Type material (holotype by original designation) in NWAU, not examined.

= Tarsozeuzera fuscipans - Yakovlev, 2004b: 342. An incorrect subsequent spelling of Tarsozeuzera fuscipars (Hampson, 1892).

DISTRIBUTION: N. India, Myanmar, Laos, Borneo, Vietnam, Thailand, China (Yunnan, Hubei) [Gaede, 1933; Arora, 1976; Barlow, 1982; Yakovlev, 2004b-c; 2014ú.
MATERIAL EXAMINED. China: $1 \sigma^{\gamma}$, China, Yunnan centr., Ailao Mts., $3500 \mathrm{~m}$ [probably sic!], 09.1999, leg. Wang \& Li (MWM); $1 \sigma^{7}$, China, Hubei, Dabie Shan, $20 \mathrm{~km}$ N Fengshan, $30^{\circ} 58 \mathrm{~N} / 115^{\circ} 26 \mathrm{E}, 12^{\prime \prime} 27$ July 2006, $1200 \mathrm{~m}$, leg. Siniaev (MWM). Myanmar: $1 \sigma^{7}$, S. Burma, Tenasserim, Khao Yai, 600"1100 m, September 1995, leg. Steinke \& Lehman (MWM). Thailand: 2 $\sigma^{7} \sigma^{7}$, Thailand, Changwat Nan, $15 \mathrm{~km} \mathrm{~N}$ of Bo Luang, 1000m, 7.04. 1998, leg. Csovari \& Steger (MWM); $1 \sigma^{7}$, Thailand, Changwat Chiang Mai, Mt. Doi Inthanon, NP, $39 \mathrm{~km}$ road N of Chom Thong, 1820 m, 1-3.06.1998, leg. Dr. Ronald Brechlin (MWM); 1 \%", Thailand, Changwat Nan, $22 \mathrm{~km} \mathrm{~N}$ of Bo Luang, 1120m, 2930.06.1998, leg. Csabo \& Soos (MWM); $1 \sigma^{7}$, N. Thailand, Lampang Prov., Mulang Pan distr., Jae Som Sub env., Baon PhayaWang., 31.0720.08.2011, leg. G. Grigoriev (RYB). India: 1 ○', India, Darjeeling, 5 km N Rambi, 900 m, 20"30.07.1990, leg. W. Thomas (MWM); $1 \sigma^{\top}$, N. India, Arunachal-Pradesh Prov., Passighat distr., 1516.07.2010, $630 \mathrm{~m}, 28^{\circ} 08^{\prime} \mathrm{N} / 9^{\circ} 15^{\prime} \mathrm{E}$, leg. G. Bretschneider (RYB). Vietnam: $1 \sigma^{\top}$, Vietnam, Thai Nuygen, Mo Ba, 06.2009 (MWM); $2 \sigma^{\top} \sigma^{\top}$, Vietnam, Kon Tum prov., Sa Thai distr., Chu Mon Ray N.[ational] P. [ark], Bargok Comm, N14²5'; E 107²4', 20.03.2012, 680 m, leg. V. Zolotuhin (RYB). Laos: $1 \sigma^{\top}$, Saysomboune prov., Phou Khao Khouay, $18^{\circ} 54^{\prime} \mathrm{N} / 103^{\circ} 08^{\prime} \mathrm{E}$, 2529.10.2008, $780 \mathrm{~m}$, Siniaev leg. (RYB).

HOST: Gliricidia Kunth. [Barlow, 1982].

\section{Tarsozeuzera kochi (Semper, 1896)} Figs 16-18, 29, 33.

Zeuzera kochi Semper, 1896: 439-440.

TYPE LOCALITY: Mittel Luzon.

TYPE MATERIAL (61 syntypes) in Senckenberg Mus., Frankfurt and CMNH.

DISTRIBUTION: Philippines.

MATERIAL EXAMINED: 1 ๙, Philippinen, Panay, Sibalom, Bontol, 9"11.12.1991, leg. Cerny (MWM); $3 \sigma^{7} \sigma^{7}$, the Philippines, Panay, Aklan, $13 \mathrm{~km} \mathrm{~S}$ Libicao, $100 \mathrm{~m}, 11^{\circ} 24 \mathrm{~N} / 122^{\circ} 18 \mathrm{E}$, June 2009, J.H. Lourens leg. (MWM); 1 ๙ै, Philippinen, Panay, Mt. Malindog, Aklan, 600"800 m, May 1997 (MWM); $1 \sigma^{7}$, Philippinen, Babuyan (N of Luzon), 1960N / 121 ${ }^{\circ} 95 \mathrm{E}$, July 2000 (MWM); $1 \sigma^{7}$, Philippinen, Negros, Mt. Kanlaon, 03.1997 (MWM); $2 \sigma^{7} \sigma^{7}$, Philippinen, Palawan, Magcasaw, Mainit Brook's Point, 3"6.11.1996, leg. Bal (MWM); 1 ऽ', Philippinen, Palawan, Mt. Cleopatra, 29.11"2.12.2000, leg. D. Mohagan (MWM); $1 \sigma^{7}$, Philippinen, Mindanao, Bukidnon, NW Maramag Mt., $1450 \mathrm{~m}$, 30.12.1991, leh. Cerny (MWM); $1 \sigma^{T}$, Philippinen, Leyte, Mt. Balocaue, Mahaplag, 18"29.12.2000 (MWM); $19 \sigma^{7} \sigma^{7}, 7$ 오, Philippinen, Negros, Mt. Canlaon, W-Route via Mambucal, 600 m, 10.1996 (MWM); $1 \sigma^{7}$, the Philippines, NW Leyte, Lake Damao, $650 \mathrm{~m}, 11^{\circ} 04 \mathrm{~N} / 124^{\circ} 42 \mathrm{E}, 29^{\prime \prime} 30.06 .2006$, leg. J.H. Lourens (MWM); $1 \mathrm{O}^{7}$, the Philippines, E. Luzon, Sirra Madre Mts, $100 \mathrm{~m}, 5 \mathrm{~km} \mathrm{~N}$ Infanta, 2 April 2004, leg. J.H. Lourens (MWM); $1 \sigma^{7}$, the Philippines, Mindoro, E of Roxas Riverbad, $12^{\circ} 42 \mathrm{~N} / 121^{\circ} 20 \mathrm{E}, 50 \mathrm{~m}, 7$ Jan. 2005, leg. J.H. Lourens (MWM); $25 \sigma^{\top} \sigma^{\top}, 3$ 우, Ins. Philipp. (MNHS).

HOST: Gliricidia Kunth. [Semper, 1896].

NOTES. Specimens from Indonesia, Sulawesi (1 $\sigma^{r}$, Dumoga-Bone; 1 ㅇ, Palu, Mt. Rangkunau in BMNH) reported by Schoorl [1990: 158], probably new species.

Tarsozeuzera livingstoni Yakovlev, 2006 Figs 19-21, 30, 34.

Yakovlev, 2006: 211

TYPE LOCALITY: Rep. Pop. Congo, Dimonica.

TYPE MATERIAL (holotype) in MNHN, examined.

DISTRIBUTION: Congo, Cameroon, Cote d'Ivoire, Malawi, Kenya, Tanzania, Zimbabwe, Zambia [Yakovlev, 2011; Yakovlev, Murphy, 2013].

MATERIAL EXAMINED: $2 \sigma^{\top} \sigma^{7}$, Zimbabwe, Bulawayo, Shangani, Naletale, 20.12.2011, leg. Snizek (MWM); $1 \sigma^{7}$, Zimbabwe, S of Harare, Beatrice, Ngezi, 10.12.2011, Snizek leg. (GenPR Heterocera MWM: 32.918); 2 o $\sigma^{7}$, Zambia, Kafue National Park, 1210 m, January 2010, R. Beck \& R. Wanninger leg. (MWM); 1 "7, 


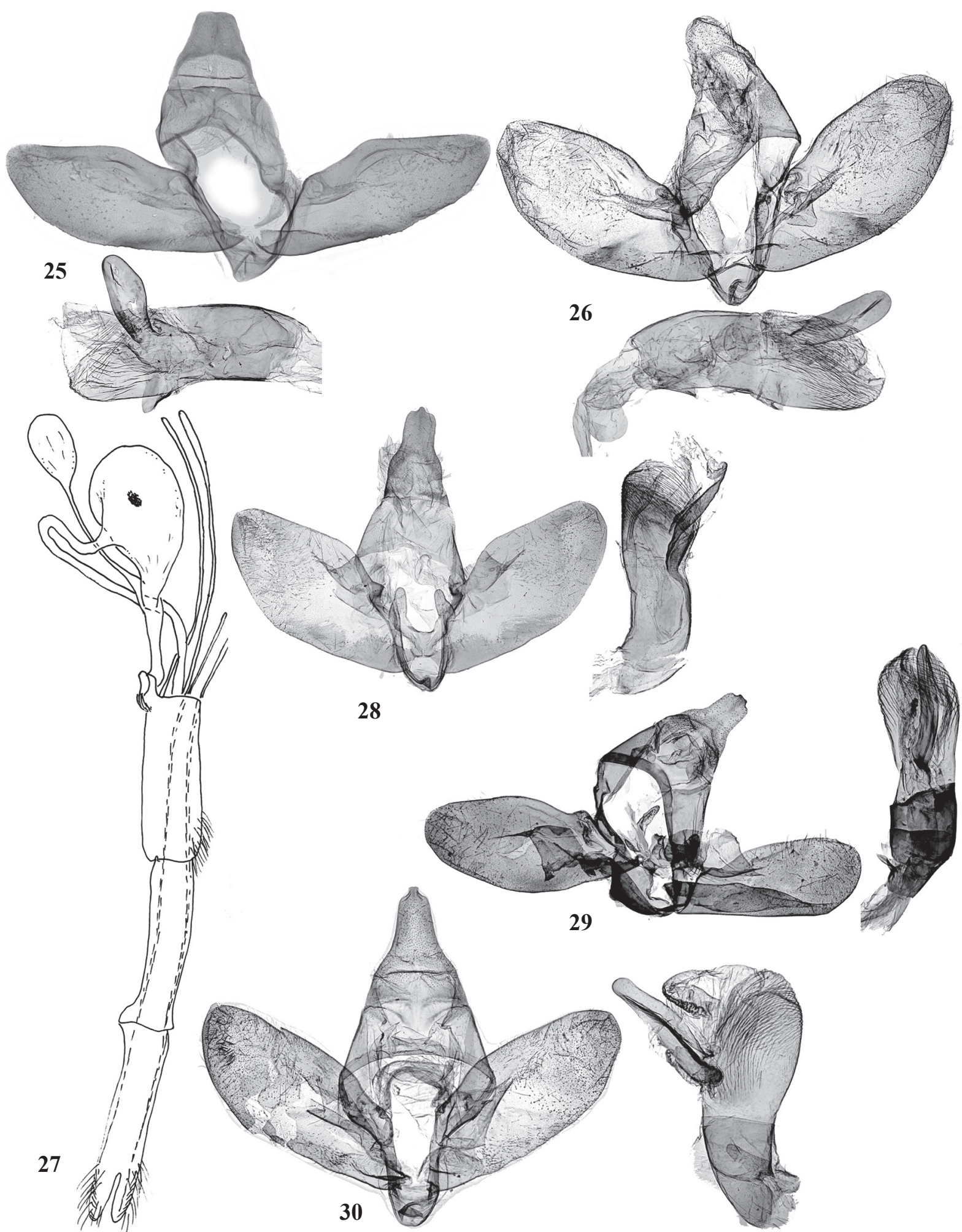

Figs 25-30. Genitalia of Cecryphalini: 25 - C. helenae, $0^{7}$, Morocco, Ait-Ou-Mribete (GenPr Heterocera MWM: 32.914); 26 - C. strix, $\sigma^{7}$, Mongolia, Bayan-Khongor aimak, Oase Echin-Gol (GenPr Heterocera MWM: 32.913); 27 - C. strix, , Turkmenistan (figured by Kozhantshikov); 28 - T. fuscipars, $\mathrm{O}^{7}$, Thailand, Changwat Nan, $15 \mathrm{~km} \mathrm{~N}$ of Bo Luang (GenPr Heterocera MWM: 32.916); 29 — T. kochi, $\sigma^{7}$, Luzon (GenPr Heterocera MWM: 32.915); 30 - T. livingstoni, $\sigma^{7}$, Zimbabwe (GenPr Heterocera MWM: 32.919).

Рис. 25-30. Гениталии Cecryphalini: 25 - C. helenae, O, Morocco, Ait-Ou-Mribete (GenPr Heterocera MWM: 32.914); 26 - C. strix, ○', Mongolia, Bayan-Khongor aimak, Oase Echin-Gol (GenPr Heterocera MWM: 32.913); 27 - C. strix, + , Turkmenistan (puc. И. Кожанчикова); 28 - T. fuscipars, $O^{7}$, Thailand, Changwat Nan, $15 \mathrm{~km} \mathrm{~N}$ of Bo Luang (GenPr Heterocera MWM: 32.916); 29 - T. kochi, $0^{7}$, Luzon (GenPr Heterocera MWM: 32.915); 30 - T. livingstoni, O’, Zimbabwe (GenPr Heterocera MWM: 32.919). 
Kenya, Strasse Nairobi-Mombasa, Tsavo National Park, Salaga Lodge, 550 m, 7"8.04.2009, leg. A. Puchner (GenPR Heterocera MWM: 32.919); 1 $\sigma^{7}$, Elfenbeinkoste, San Pedro, 1"4.12.1977, leg. Politzar (GenPR Heterocera MWM: 32.920); 1 ऊ', Cameroon, Ebogo, 23"29.11.1992, Th. Bouyer (MRAC); 1 o', Cameroon, Efulen, 16 Nov. 1922, H.L. Weber (CMNH); 1 \%, Congo, Sankuru, Katoko-Kombe, 17.12.1951, Dr. Fontaine (MRAC); $2 \sigma^{7} \sigma^{7}$, Uele, Paulis, 31.12.1959, Dr. M. Fontaine (MRAC); $3 \sigma^{\top} \sigma^{7} \sigma^{\top}$, Leopoldville, 27.08.1953, Dr. M. Fontaine (MRAC); 1 య', Sankuru, 14.06.1952, Dr. M. Fontaine (MRAC); $1 \sigma^{7}$, Mwenewisi Forest, Karonga distr., N. Malawi, 2200 ft., 25.02.2001, leg. R.J. Murphy (RMM); 1 ㅇ, Chimaliro FR, Kasungu Distr., C. Malawi, $4200 \mathrm{ft}$., 26.02.1998, leg. R.J. Murphy (RMM); 1 \%, same locality, 29.11.1997, leg. R.J. Murphy (RMM); 1 क, same locality, 31.01.1998, leg. R.J. Murphy (RMM); 1 o, same locality, 23.02.1998, leg. R.J. Murphy (RMM); 1 , same locality, 6.02.2000, leg. R.J. Murphy (RMM); 1 $\sigma^{7}$, Tanzanie, Rukwa Reg., Kalambo Forest Reserve, 1577 m,
08²2.241'S / 031 ${ }^{\circ} 14.573$ 'E, 31.10.2007, leg. Ph. Darge (GenPR Heterocera MWM: 33.175)

TAXONOMICAL NOTES. In CMNH a male specimen of $T$. livingstoni from Cameroon (Efulen), handwritten by H. Clench as the holotype of"Tacticista (n.g.) subhyalinata Clench". This taxon was not described and is considered a unpublished "manuscript name".

Tarsozeuzera miklukhomaklayi Yakovlev, 2011 Figs 22, 35.

Yakovlev, 2011: 64.

TYPE LOCALITY: Dutch New Guinea, Humboldt Bay Dist., Kroisni.

TYPE MATERIAL (holotype) in BMNH, examined.

MATERIAL EXAMINED: $1 \sigma^{\top}$ (holotype), Dutch New Guinea, Humboldt Bay Dist., Kroisni, 12.06.1937. (BMNH).

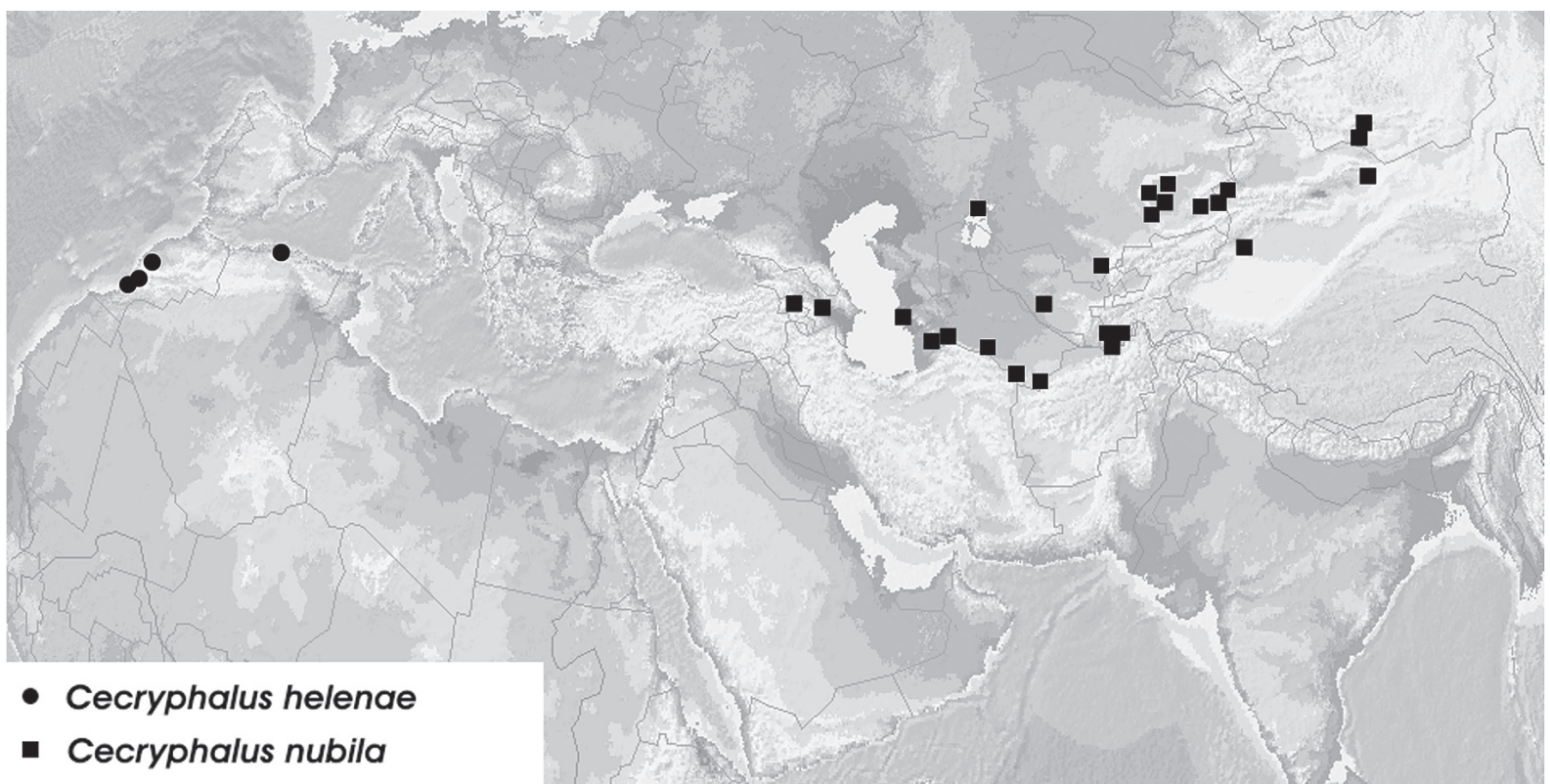

Fig. 31. Distributional map of Cecryphalus

Рис. 31. Карта распространения Cecryphalus.
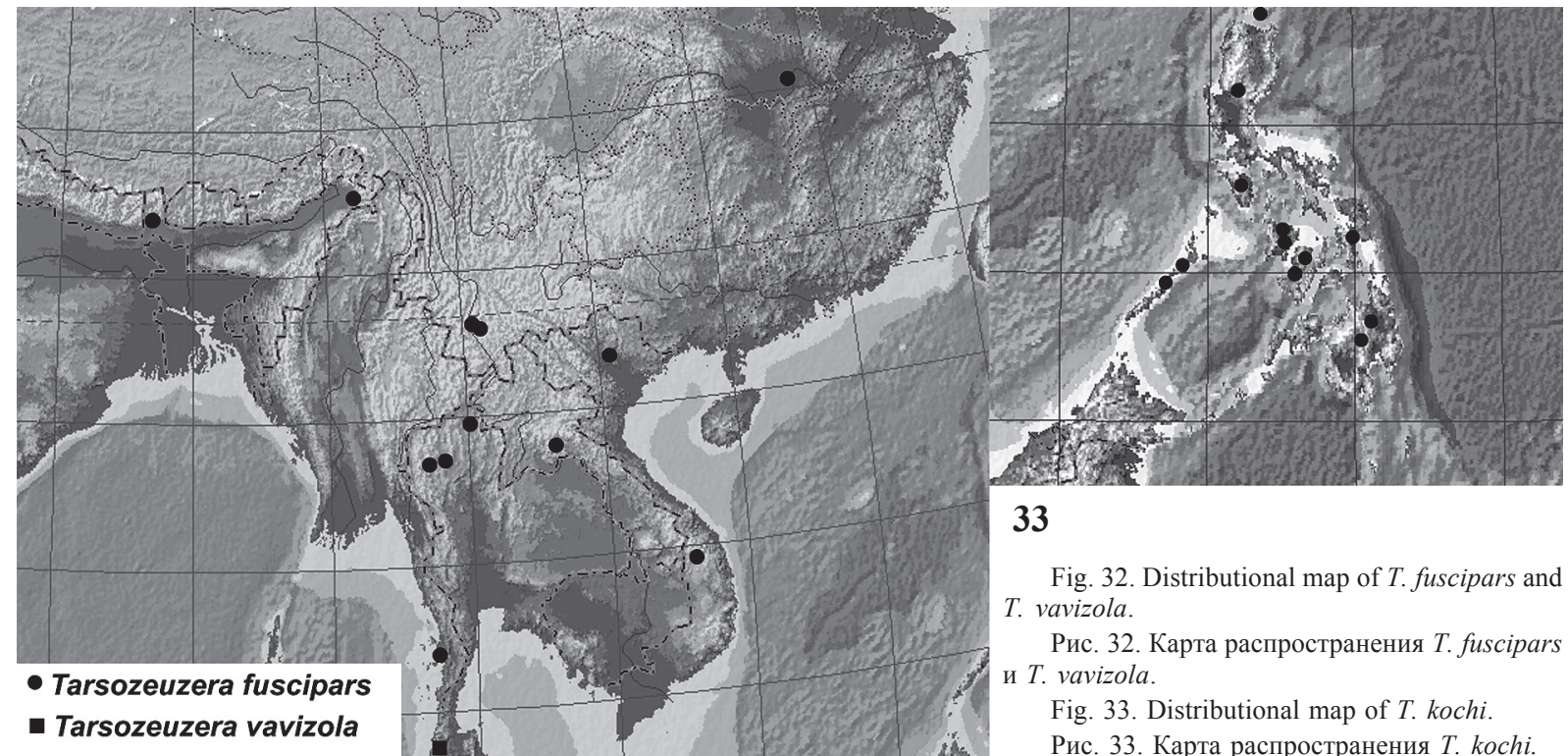

\section{3}

Fig. 32. Distributional map of T. fuscipars and T. vavizola.

Рис. 32. Карта распространения T. fuscipars и $T$. vavizola.

Fig. 33. Distributional map of T. kochi.

Рис. 33. Карта распространения T. kochi. 
Tarsozeuzera ustjuzhanini Yakovlev, 2011

Figs 23, 34.

Yakovlev, 2011: 64.

TYPE LOCALITY: S. Afrika, Kwazulu Natal, Vernon-Crookes NP, $60 \mathrm{~km}$ SW Durban.

TYPE MATERIAL (holotype) in MWM, examined.

DISTRIBUTION. South Africa (Kwa Zulu-Natal Prov.).

MATERIAL EXAMINED: $10^{\gamma}$, RSA, EC [Eastern Cape], nr. Port St. Johns, 13.12.2002, leg. Legrain (coll. Legrain).

Tarsozeuzera vavizola Yakovlev, 2006 Figs 24, 32.

Yakovlev, 2006: 211.

TYPE LOCALITY: $20 \mathrm{~km}$ O. Krabi, S. Thailand TYPE MATERIAL (holotype) in ZSM, examined. DISTRIBUTION: S. Thailand.

ACKNOWLEDGMENTS. The authors express their gratitude to the colleagues: Didier Van den Spiegel, Alice Buset (Tervuren), Jurate and Willy De Prins, Wolfram Mey (Berlin), Axel Hausmann (Munich), Igor Kostyuk (Kiev), Joel Minet (Paris), Sergey Sinev (St. Petersburg), Raymond Murphy (Mzuzu), Manfred Ströhle (Weiden), Armin Hauenstein (Untermünkcheim) for the possibility to examine the materials on Cossidae at MRAC, MNKB, ZSM, ZMKU, MNHN, ZISP, RMM, MSW, and AHU. We are indebted to the BMNH Council of Trustees for kindly granting me the permission to publish images of type specimens preserved in the Natural History Museum. The authors are grateful to Anna Ustjuzhanina (Tomsk) for the help in translation of the paper. The English text was reviewed by Prof. Boris Kondratieff (Fort Collins) We also express our gratitude to H. Sulak (Munich) and L. Aarvik (Oslo) for making the photo and to O. Bidzilya (Kiev) for the assistance in making genital preparations. The financial support for this study was provided by the grant No. 18-0400440 A from the Russian Foundation for Basic Research.

\section{References}

Arora G.S. 1976. A taxonomic revision of the Indian species of the family Cossidae (Lepidoptera) // Records of the Zoological Survey of India. Vol.69. No.1-4. P.1160.

Austaut J.-L. 1897. Notice sur quelques cossided nouveaux // Le Naturaliste. Ann.19 (Sér. 2. Ann. 11). P.44-45.

Barlow H.S. 1982. An introduction to the Moths of South East Asia. Kuala Lumpur: Malayan Nature Society. 305 pp.

Gaede M. 1933. Cossidae // A. Seitz (Ed.) Die Gross-Schmetterlinge der Erde. Bd.10. Spinner und Schwärmer des indo-australischen Gebiets. Stuttgart: Alfred Kernen Verlag. S.809-823.

Grum-Grshimailo Gr. 1895. Lepidoptera Palaearctica nova // Horae Societatis Entomologicae Rossicae. Vol.29. P.290-293.

Dalla Torre K.W., von. 1923. Cossidae // Lepidopterorum Catalogus. Berlin. 63 pp.

Daniel F. 1969. Bombyces et Sphinges III. Ergebnisse der zoologishen Forschungen von Dr. Z. Kaszab in der Mongolei (Lep.) // Reichenbachia. Bd.11. S.265-277.

Daniel F. 1970. 200. Bombyces et Sphinges. 4. Ergebnisse der zoologische Forschungen von Dr. Z. Kaszab in der Mongolei // Reichenbachia. Bd.13. No.19. S.193-203.

Freina J.J., de, Witt T.J. 1990. Cossidae // Die Bombices und Sphinges der Westpalaearktis. Bd.2. München. S.9-41.

Hampson G.F. 1892. Moths. Vol.1// The fauna of British India including Ceylon and Burma. The Hague: Dr. W. Junk b.v. Publishers. 527 pp.

Hua B., Chou I., Fang D., Chen S. 1990. The Cossid fauna of China (Lepidoptera, Cossidae). Yangling, Shaanxi. 147 pp.

Nieukerken van E.J., Kaila L., Kitching I.J., Kristensen N.P., Lees D. C., Minet J., Mitter C., Mutanen M., Regier J.C., Simonsen T.J., Wahlberg N., Yen S.-H., Zahiri R., Adamski D., Baixeras J., Bartsch D., Bengtsson B.A., Brown J.W., Bucheli S.R., Davis D.R., De Prins J., De Prins W., Epstein M.E., Gentili-Poole P., Gielis C., Hättenschwiler P., Hausmann A., Holloway J.D., Kallies A., Karsholt O., Kawahara A., Koster J.C., Kozlov M.V. Lafontaine J.D., Lamas G., Landry J.-F., Lee S., Nuss M., Park K.T., Penz C., Rota J., Schmidt B.C., Schintlmeister A., Sohn J.C., Solis M.A., Tarmann G.M., Warren A.D., Weller S.,

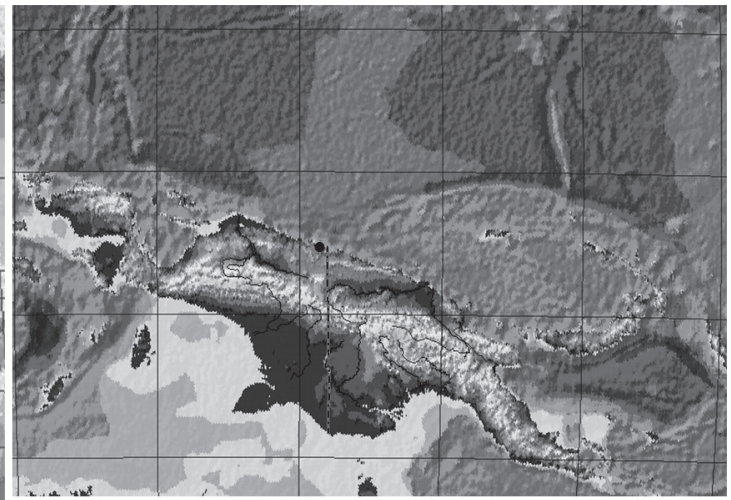

35

Fig. 34. Distributional map of $T$. livingstoni and $T$. ustjuzhanini.

Рис. 34. Карта распространения $T$. livingstoni и $T$. ustjuzhanini.

Fig. 35. Distributional map of T. miklukhomaklayi.

Рис. 35. Карта распространения T. miklukhomaklayi 
Yakovlev R.V., Zolotuhin V.V., Zwick A. 2011. Order Lepidoptera Linnaeus, 1758 // Zootaxa. Vol.3148. P.212-221.

Rungs Ch. 1942. III. Notes de lepidopterologie Marokaine (XI) Additions a faune du Maroc: Lepidopteres des regions Sahariennes // Bull. Soc. Sc. Nat. Maroc. No. 22. P. 177.

Rungs Ch.E.E. 1979. Catalogue raisonné des Lépidoptères du Maroc // Inventaire Faunistique et observations écologiques. T.1. Rabat. P.28-32.

Schoorl J.W. 1990. A phylogenetic study on Cossidae (Lepidoptera: Ditrysia) based on external adult morphology // Zoologische Verhandelingen. Vol. 263. 295 p. $1 \mathrm{t}$.

Seitz A. 1912. Die Gross-Schmetterlinge der Erde. Bd. 2: Die Palaearktischen Spinner \& Schwärmer. Stuttgart. 479 S.

Semper G. 1896-1902. Die Schmetterlinge der Philippinischen Inseln. Beitrag zur indo-malayischen Lepidopteren-Fauna. Zweite Abtheilung. Die Nachtfalter - Heterocera // Reisen im archipel der Philippinen. Wiesbaden: C. W. Kreidel. S. 381728. https:// doi.org/10.5962/bhl.title.8793.

Sheljuzhko L. 1913. V. Eine neue Zeuzera aus Transkaukasien // Deutsche Entomologische Zeitschrift, Iris. Bd.27. S.21-22.

Staudinger O. 1895. Ausser den im Vorstehenden beschriebenen Arten vom eigentlichen Tibet beschreibe ich noch aus dem Inneren Asiens stammende Arten // Deutsche Entomologische Zeitschrift, Iris. Bd.8. S.300-343.

Yakovlev R.V. 2004a. Carpenter-moths (Lepidoptera, Cossidae) of Mongolia // Euroasian Entomological Journal. Vol.3. No.3. P.217-224.
Yakovlev R.V. 2004b. Cossidae of Thailand. Part I. // Atalanta. Bd.35. No.3/4. P.335-352.

Yakovlev R.V. 2004c. New data about Carpenter-Moths (Cossidae) of China // Atalanta. Bd. 35. No.3/4. P.353-356.

Yakovlev R.V. 2006. New Cossidae (Lepidoptera) from Asia, Africa and Macronesia // Tinea. Vol.19. No.3. P.188-213.

Yakovlev R.V. 2011a. [A brief Review of the Genus Cecryphalus Schoorl, 1990 (Lepidoptera, Cossidae)] // Euroasian Entomological Journal. Vol.10. No.1. P.19-21 [in Russian].

Yakovlev R.V. 2011b. Catalogue of the Family Cossidae of the Old World (Lepidoptera) // Neue Entomologische Nachrichten. Bd.66. S.1-130.

Yakovlev R.V. 2014. Descriptions of three new species of Cossidae (Lepidoptera) from Vietnam, with an updated annotated checklist // Zootaxa. Vol.3802. No.2. P.240-256.

Yakovlev R.V. 2015. Cossidae (Lepidoptera) of Mongolia // Check List. Vol.11. No.5. Article 1736, doi: http://dx.doi.org/10.15560/ 11.5.1736

Yakovlev R.V., Dubatolov V.V. 2013. Distribution of carpentermoths (Lepidoptera, Cossidae) in palaearctic deserts // Zoologicheskii Zhurnal. Vol.92. No.6. P.682-694 [in Russian].

Yakovlev R.V., Murphy R.J. 2013. The Cossidae (Lepidoptera) of Malawi with descriptions of two new species //Zootaxa. Vol.3709. No.4. P.371-393. DOI: 10.11646/zootaxa.3709.4.5.

Yakovlev R.V., Witt T.J. 2016. Carpenter-moths of Turkmenistan (Lepidoptera: Cossidae) // Biological Bulletin of Bogdan Chmelnitskiy Melitopol State Pedagogical University. Vol.6. No.3. P.164-173. 